\title{
Prevalência de tabagismo e fatores associados em área metropolitana da região Sul do Brasil*
}

\author{
Prevalence of smoking and associated factors in a metropolitan \\ area of southern Brazil
}

\author{
Leila B. Moreira, Flávio D. Fuchs, Renan S. Moraes, Markus \\ Bredemeir, Sívia Cardozo
}

Departamento de Farmacologia da Universidade Federal do Rio Grande do Sul,-Brasil (L. B. M. F. D. F.); Unidade de Farmacologia Clinica do Hospital de

Clínicas de Porto Alegre - Brasil (L. B. M., F. D. F., R. S. M. M. B., S. C.)

\begin{abstract}
Com o objetivo de avaliar a prevaléncia de tabagismo em Porto Alegre, RS, Brasil, e os fatores associados, executouse estudo observacional, de delineamento transversal e base populacional. Através de amostragem aleatória proporcional, por estágios múltiplos e conglomerados, selecionaram-se 1.091 individuos, a partir de 18 anos, que responderam a um questionário, em entrevista domiciliar. Aferiu-se o hábito de fumar através de perguntas dirigidas ao tipo de fumo, freqüência e tempo de exposiçăo. A prevalencia fol de $34,9 \%$ (IC 31,9 - 37,8), sendo de $41,5 \%$ (IC $38,5-44,4)$ entre os homens e $29,5 \%$ (IC 26,8 - 32,2) entre as mulheres. O inicio fol, em médja, aos $16( \pm 5,6)$ e 17,8 $( \pm 6,7)$ anos, com moda de 15 e 14 anos, respectivamente. Os homens fumavam $19,0 \pm 14,0$ clgarros por dia e as

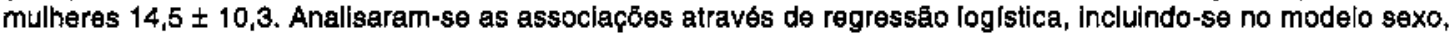
Idade, educação, renda, qualificação profissional e consumo de álcool. O hábito de fumar foi mais freqüente entre os homens, Indivíduos de menor nivel socioeconómico, dos 30 aos 39 anos, e entre os usuários de bebidas alcóblicas. Conclui-se que o tabagismo é freqũente em Porto Alegre, constituindo-se problema de saúde pública similar ao referido pela literatura. O consumo de álcool deve estar associado ao fumo por serem ambos comportamentos de risco, com determinantes comuns.
\end{abstract}

Fumo, epidemiologia. Fatores de risco.

\section{Introduçāo}

O tabaco é empregado nas Américas há milhares de anos, sob diversas formas e com propósitos culturais e sociais. Nas sociedades indígenas sulamericanas é parte essencial de ritos religiosos e elemento básico para os chefes espirituais, como mecanismo para exercer sua autoridade e conservar a credibilidade. Os exploradores europeus tiveram seu primeiro contato com o tabaco nas Antilhas, quando os nativos ofereceram as folhas da planta a Crist 6 vāo Colombo. Diferentemente dos efeitos tóxi$\cos$ e organolépticos buscados pelos índios, o consumo do tabaco objetiva, nas sociedades modernas da América Latina, cada vez mais o prazer social decorrente de seus efeitos estimulantes, com a instalaçăo da dependência a longo prazo e as consequiências crônicas para saúde". Desde o início do século, a produção de cigarros no Brasil se inseriu em uma linha de produçăo industrial, com o estímulo do mercado consumidor. Em decorrência, o hábito de fumar associou-se a um padrăo de vida mais elevado, difundindo-o amplamente ${ }^{\mathbb{R}}$.

O fumo é importante causa de perda de saúde. Está associado ao desenvolvimento de doenças respiratorias, cardiovasculares e

*Pesquisa subvencionada pelo Fundaçāo de Amparo a Pesquisa do Rio Grande do Sul/FAPERGS (Processo 0971/89) e pelo Conselho Nocional de Desenvolvimento Cientfico e Tecnológico/CNPq (Processo 500550/89-1).

Separatas/Reprints: Flavio D. Fuchs - Departamento de Farmacologia da Universidade Federal do Rio Grande do Sul R. Ramiro Barcelos, 2350, sala 947 - 90035-003 - Porto Alegre, RS - Brasil

Recebido em 24.8.1994. Aprovado em 29.11.1994. 
neoplasias. A mortalidade geral é duas vezes maior nos fumantes quando comparados aos não-fumantes ${ }^{3}$. Constitui-se um dos fatores de risco maiores para doença coronariana ${ }^{3,14}$. Juntamente com as neoplasias, as doenças cardiovasculares representam a maior causa de morte no Rio Grande do $\mathrm{Sul}^{10} \mathrm{e}$ em grandes cidades do Brasit20,21,23.

Apesar de ser importante fator de risco, cuja remoçāo repercute significativamente na redução da morbidade e mortalidade, os dados de prevalência de tabagismo na população geral brasileira são esparsos. Nos poucos estudos populacionais, as estimativas oscilam entre 32 e $42 \%{ }^{1,10,22,24,26}$. É maior entre os homens, da $3^{\mathbf{H}}$ à $6^{a}$ década de vida e posição socioeconômica inferior ${ }^{10,11,22,24}$. A associação inversa do tabagismo com nível socioeconômico também é observada internacionalmente ${ }^{5,13}$.

O presente estudo descreveu a prevalência contemporânea de tabagismo em Porto Alegre, Rio Grande do Sul, permitindo compará-la com a da década de 70 . Avalia, também, a associação do hábito de fumar com diversos fatores biológicos e demográficos.

\section{Material e Método}

Foi executado um estudo observacional, analítico, de delineamento transversal e de base populacional. O processo de amostragem aleatória proporcional deu-se por estágios múltiplos e por conglomerados, representando a população adulta urbana de Porto Alegre. Nos domicilios sorteados foram incluídos todos os moradores com 18 anos ou mais.

Dos 1.188 selecionados, foram efetiva. mente entrevistados 1.091 indivíduos $(91,9 \%)$. Esta amostra, calculada previamente para um estudo sobre hipertenção arterial, conferiu uma precisão da estimativa de prevalência de tabagismo de $\pm 3 \%$, para um intervalo de confiança de $95 \%$. As perdas deveram-se, em sua maioria, a recusa após pelo menos 3 tentativas.

Os dados foram coletados em visita domiciliar, através de questionário contendo questōes referentes a dados pessoais, socioeconômicos, uso de fármacos e álcool, medidas antropométricas e de pressão arterial. O hábito de fumar foi aferido através de perguntas dirigidas ao tipo de fumo, frequência e tempo de exposiçāo. $O$ controle de qualidade foi feito pela revisita a $10 \%$ dos entrevistados.
A presente comunicação restringe-se ao tabagismo, não sendo, portanto, apresentados os dados relacionados à pressăo arterial. As seguintes variáveis foram consideradas para análise das associaçōes:

-fumo: variável dependente dicotômica, categorizada em não-fumante e fumante, que incluiu fumantes atuais e ex-fumantes, a partir de 4 unidades por semana, indepedentemente do tipo de fumo (cigarro, palheiro, charuto, cachimbo).

-sexo

-idade: variável quantitativa categorizada em 4 níveis: 18 a 29, 30 a 39,40 a 49,50 a 88 anos.

-consumo de álcool: variável qualitativa dicotômica; foram considerados expostos os indivíduos que faziam uso de bebidas alcó́licas no momento ou no passado, independentemente da quantidade.

-cor: variável qualitativa, dicotômica; branco e não-branco, determinada a partir da observaçăo do entrevistador.

-renda "per capita" mensal: em salários mínimos, correspondente à soma das rendas individuais dividida pelo total de moradores do domichio. Foi categorizada em três níveis de salários-mínimos: menos de 2,2 a menos de $4 \mathrm{e}$ 4 ou mais.

-escolaridade: qualificada em 3 categorias: 0 a 5,6 a 11 anos de escolaridade e curso superior (completo ou não).

-qualificaçăo profissional: considerando otipo e a similitude das atjvidades ${ }^{17.28}$, a qualificação profissional foi classificada nos seguintes grupos:

I: graduados ou estudantes de nivel superior, educadores, intelectuais e dirigentes.

II: proprietários de indústrias, comércios ou negócios agrários.

III: trabalhadores de qualificação intermediária.

IV: trabalhadores não-qualificados.

$\mathrm{V}$ : atividades mal-definidas e donas-de-casa.

Análise estatística: a distribuiçăo das principais características estudadas são apresentadas pelas freqüências, média e desvio-padrão (DP) e proporçōes, com seus intervalos de confiança de $95 \%$ (IC). $\mathrm{Na}$ avaliação da significância das razōes de prevalência brutas empregou-se o Qui-quadrado e o IC ${ }^{18}$. Para analisar-se conjuntamente a associação entre os diversos fatores estudados com a prevalência de tabagismo empregou-se modelo de regressão logística (SPSS) ${ }^{19}$. A seleção das variáveis explanatórias partiu do modelo teórico dos autores, 
incluindo, pelo método de entradas forçadas, aquelas cujas razões de prevalência brutas mostraramse estatisticamente significativas $(\mathrm{P}<0,05)$ : sexo, idade, consumo de bebidas alcoólicas, renda, escolaridade e qualificação profissional.

Aspectos éticos: o projeto está dentro da categoria II da regulamentaçāo de pesquisa no homem no Brasil (risco mínimo), pois envolve somente questionários e medidas antropométricas e de pressāo arterial ${ }^{5}$. Foi garantido aos entrevistados o sigilo e solicitado o consentimento verbal para realização das entrevistas.

\section{Resultados}

A comparação de variáveis demográficas aferidas ao presente estudo, como sexo, idade e renda, com as fornecidas pelo censo de $1991^{15}$ e PNAD-9016, confirmam que a amostra é representativa de Porto Alegre. A idade na amostra variou de 18 a 88 anos, com média de 41,3 $( \pm 16,2)$ anos. As demais características demográficas são apresentadas na Tabela 1.

As bebidas alcoólicas eram consumidas por $67,6 \%$ dos indivíduos, $8,2 \%$ eram ex-bebedores e $24,1 \%$, abstêmios. A prevalência global de tabagismo foi de $34,9 \%$ (IC $31,9-37,8$ ), sendo apresentada, por sexo, ла Tabela 2 . Seu

Tabela1. Características demográficas da amostra. Porto Alegre, 1991.

\begin{tabular}{llcr}
\hline Variável & Condiçąo & $\mathrm{N}$ & \multicolumn{1}{c}{$\%$} \\
\hline Sexo & feminino & 600 & 55 \\
& masculino & 491 & 45 \\
Idade & 18-29 anos & 301 & 27,6 \\
& $30-39$ anos & 225 & 20,6 \\
& $40-49$ anos & 190 & 17,4 \\
& $50-88$ anos & 375 & 34,4 \\
Renda* & 4 ou mais SM & 467 & 42,8 \\
& $2-3,99$ SM & 278 & 25,5 \\
& menos de 2 SM & 346 & 31,7 \\
Escolaridade & mais de 11 anos & 251 & 23,0 \\
& $6-11$ anos & 479 & 43,9 \\
& $0-5$ anos & 361 & 33,1 \\
Profisão** & grupo I & 228 & 20,9 \\
& grupo II & 55 & 5,0 \\
& grupo III & 204 & 18,7 \\
& grupo IV & 332 & 30,4 \\
& grupo V & 269 & 24,7 \\
& branca & 962 & 88,2 \\
Cor*** & năo-branca & 126 & 11,8 \\
\hline
\end{tabular}

"Renda per capita, em salário-mínimos.

"Ver definição em métodos; 3 mulheres não tiveram a profissāo registrada.

**3 mulheres nāo tiveram a cor registrada. início ocorreu, em média, aos $16( \pm 5,6)$ anos para os homens e $17,8( \pm 6,7)$ anos para as mulheres, com moda, de 15 e 14 anos, respectivamente. Como pode ser visto nas Tabelas 3 e 4, maior proporção dos primeiros fumam 20 ou mais cigarros por dia, concentrando-se esta diferença a partir dos 40 anos. O número médio de cigarros ou similares consumidos por dia foi de 19,2 ( $\pm 13,9)$ entre os homens e $14,5( \pm 10,3)$ entre as mulheres. Quase todas as unidades correspondiam a cigarros $(19,0 \pm 14,0$ entre os homens e $14,5 \pm 10,3$ entre as mulheres).

As razões de prevalência brutas de tabagismo entre as diversas características avaliadas no inquérito são apresentadas na Tabela 5. Com exceção da cor, todas as demais evidenciaram associação estatisticamente significativa com a prática de fumar. Na Tabela 6 encontram-se as razōes dos produtos cruzados resultantes da análise de regressão logística. A renda mostrou forte tendência a associação inversa. Manteve-se a associaçāo significativa com todas as demais variáveis incluídas no modelo, embora menos intensa com os indicadores de nível socioeconômico.

\section{Discussão}

O fumo é fator de risco ainda com alta prevalência na população adulta de Porto Alegre. Situa-se entre os observados no final da década de 80 em São Paulo e Araraquara, mais recentemente ${ }^{22}(37,9 \%$ e $32,8 \%$, respectivamente) e acima da descrita nos Estados Unidos $(25,7 \%$, em 1991) . Também se aproxima do limite superior para a América Latina, segundo inquérito realizado pela OPS em 1971, em 8 grandes cidades latinoamericanas (21 a 40\% $)^{9}$. Dois estudos realizados em Porto Alegre, em $1987^{11}$ e em $1988^{24}$, obtiveram estimativas mais elevadas, com $50 \%$ de homens e $32 \%$ de mulheres fumantes, no primeiro, e $40,8 \%$ de tabagistas, no segundo. As diferenças provavelmente se devam aos critérios amostrais.

Há cerca de 25 anos têm-se concentrado esforços mundiais para controlar a epidemia do fumo. En 1990, a $43^{\circ}$ Assembléia Mundial da Saúde confirmou a eficácia das estratégias legais e políticas que visam a proteger contra a exposiçāo involuntária ao fumo em locais de trabalho, locais públicos 
Tabela 2. Prevale̊ncia de tabagismo (\%), por sexo, em Porto Alegre, 1991.

\begin{tabular}{lccc} 
Condição & Homens (IC) & Mulheres (IC) & Total (IC) \\
\hline Fumantes & $41,5(38,5-44,4)$ & $29,5(26,8-32,2)$ & $34,9(31,9-37,8)$ \\
Ex-fumantes & $22,6(20,1-25,0)$ & $14,2(16,2-12,1)$ & $19,0(15,7-20,2)$ \\
Náo-fumantes & $35,8(32,9-38,6)$ & $56,3(53,3-59,2)$ & $47,1(44,1 \cdot 50,0)$
\end{tabular}

e nos transportes coletivos, aumento do preço real e controle da publicidade ${ }^{y}$. Tendência à diminuiçăo do número de indivíduos fuman-

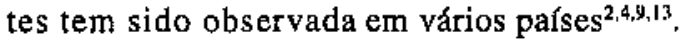

Nos últimos 20 anos, em Porto Alegre, a prevalência decresceu de $42,4 \%{ }^{1,7}$ para $34,9 \%$, significando que $17,7 \%$ dos fumantes deixaram de sê-lo, para a qual contribuíram ambos os sexos. Já em Sảo Paulo ${ }^{26}$, o decréscimo deveu-se apenas aos homens. $O$ número médio de cigarros fumados por dia é comprarável ao relatado na Inglaterra ${ }^{13}$ - 20 cigarros pelos homens e 15 pelas mulheres - , mas maior proporção, tanto de homens como de mulheres portalegrenses, consome mais de 20 cigarros por dia. Já os homens de Araraquara fumam mais que os de Porto Alegre e o inverso ocorre com as mulheres ${ }^{22}$.

$O$ início do hábito na adolescência repete o observado entre estudantes brasileiros ${ }^{27,24}$, e de outros países ${ }^{12,25}$ e justifica o desenvolvimento de programas de prevenção ao tabagismo nesta faixa etária. A distribuiçăo ao longo da iđade é compatível com os dados da literatu$\mathrm{ra}^{4,11,22,24}$. O decréscimo observado após os 40 anos pode decorrer de morte prematura e do abandono do fumo devido ao desenvolvimento de doenças associadas.

Também merece destaque a associação entre fumo e consumo de bebidas alcoólicas, outra importante causa de perda de saúde. Em estudo sobre fatores de risco para doenças não

Tabela 3. Prevale̊ncia de tabagismo (\%) em número de cigarros* consumidos por dia, segundo a faixa etária, entre os homens. Porto Alegre, 1991.

\begin{tabular}{lrrrrr}
\hline & \multicolumn{5}{c}{ Idade } \\
\cline { 2 - 6 } $\begin{array}{l}\text { Cigarros/ } \\
\text { dia }\end{array}$ & $18-29$ & $30-39$ & $40-49$ & $50-88$ & Total \\
\hline $0^{* *}$ & 60,1 & 54,6 & 60,5 & 59,4 & 58,9 \\
até 9 & 9,8 & 10,3 & 5,8 & 6,5 & 8,1 \\
$10-19$ & 13,7 & 9,3 & 4,7 & 5,8 & 8,8 \\
$20-39$ & 15,0 & 20,6 & 23,3 & 20,6 & 19,3 \\
40 & 1,3 & 5,2 & 5,8 & 7,7 & 4,9 \\
\hline
\end{tabular}

*Cigarros sem flitro, com filtro ou palheiro

"Não-fumantes ou ex-fumantes transmissíveis em Porto Alegre" soas reuniam dois ou mais fatores, sendo o fumo um dos dois mais freqüentemente acumulados.

As características sociodemográficas da população latinoamericana concorrem para a maior suscetibilidade ao tabagismo. Entre elas, destacam-se as mudanças da estrutura da população, maior urbanização, ingresso da mulher na população economicamente ativa, maior acesso à educaçăo e conseqüentemente à propaganda". Os indicadores socioeconômicos avaliados no presente inquérito apontaram maiores prevalências de tabagismo nas categorias inferiores, em concordância com o descrito em outros estudos ${ }^{4,5,10,13,22}$. Quando foi isolado o efeito da renda, permaneceu apenas uma tendência de associação inversa, inicialmente observada na análise bruta. Pode-se especular que haja restrição a aquisição de cigarros pela falta absoluta de dinheiro.

O perfil descrito no presente estudo corresponde, em geral, ao referido pela literatura. Sexo masculino, idade entre 30 e 39 anos, baixo nível socioeconômico e consumo de bebidas alcoólicas são os fatores de risco identjficados. Dada a natureza transversal do estudo, não se pode afirmar que as associaçōes nele observadas sejam de natureza causal. Assim, 0 consumo de álcool deve estar associado ao fumo por serem ambos comportamentos de risco, com determinantes comuns.

Tabela 4. Prevalecéncia de tabagismo (\%) em número de cigarros* consumidos por dia, segundo a faixa etária, entre as mulheres. Porto Alegre, 1991.

\begin{tabular}{lrrrrr}
\hline & \multicolumn{5}{c}{ Idade } \\
\cline { 2 - 6 } $\begin{array}{l}\text { Cigarros/ } \\
\text { dia }\end{array}$ & $18-29$ & $30-39$ & $40-49$ & $50-88$ & Total \\
\hline $0^{* *}$ & 67,3 & 57,0 & 67,3 & 84,5 & 71,5 \\
até 9 & 8,8 & 11,7 & 11,5 & 3,6 & 8,0 \\
$10-19$ & 8,2 & 7,8 & 8,7 & 6,8 & 7,7 \\
$20-39$ & 15,0 & 21,9 & 10,6 & 5,0 & 12,0 \\
40 ou mais & 0,7 & 1,6 & 1,9 & 0,0 & 0,8 \\
\hline
\end{tabular}

* Cigarros sem filtro, com filtro ou palheiro

** Nāo-fumantes ou ex-fumantes 
Tabela 5. Razões de prevaléncia brutas (RP) de tabagismo conforme as diferentes condiçōes de interesse. Porto Alegre, 1991.

\begin{tabular}{|c|c|c|c|}
\hline Condiģăo & & $\mathrm{RP}(\mathrm{IC})$ & $P$ \\
\hline Sexo & $\begin{array}{l}\text { ferminino' } \\
\text { masculino }\end{array}$ & $\begin{array}{c}1 \\
1,47(1,31-1,64)\end{array}$ & $<0,0001$ \\
\hline Idade & $\begin{array}{l}18.29 \text { anos } \\
30-39 \text { anos } \\
40-49 \text { anos } \\
50.88 \text { anos }\end{array}$ & $\begin{array}{c}1 \\
1,46(1,25-1,70) \\
1,19(1,00-1,44) \\
1,12(0,96-1,32)\end{array}$ & $<0,0001$ \\
\hline Renda*" & $\begin{array}{l}4 \text { ou mals SM" } \\
2-3,99 \mathrm{SM} \\
\text { menos de } 2 \mathrm{SM}\end{array}$ & $\begin{array}{c}1 \\
0,99(0,86-1,16) \\
1,20(1,06-1,37)\end{array}$ & 0,0075 \\
\hline Escolaridade & $\begin{array}{l}\text { mais do } 11 \text { anos" } \\
6.11 \text { anos } \\
0-5 \text { anos }\end{array}$ & $\begin{array}{c}1 \\
1,37(1,16-1,61) \\
1,27(1,07-1,51)\end{array}$ & 0,0003 \\
\hline Profissaxo"n* & $\begin{array}{l}\text { grupo I" } \\
\text { grupo II } \\
\text { grupo I!l } \\
\text { grupo NV } \\
\text { grupo V }\end{array}$ & $\begin{array}{c}1 \\
1,41(1,07-1,85) \\
1,39(1,14-1,69) \\
1,49(1,25-1,78) \\
1,17(0,96-1,43)\end{array}$ & 0,0001 \\
\hline Cor & $\begin{array}{l}\text { branca* } \\
\text { nāo-branca }\end{array}$ & $\begin{array}{c}1 \\
1,16(0,99-1,35)\end{array}$ & 0,0995 \\
\hline USO de Álcool & $\begin{array}{l}\text { กต̆о* } \\
\text { sim }\end{array}$ & 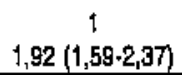 & $<0,0001$ \\
\hline
\end{tabular}

* Categoria de referéncia

** Renda per capita, em salários- minimos

** Ver métodos

\section{Referênclas Blbllográficas}

1. ACHUTTI, A. \& MEDEIROS, A. B. Hipertensăo arterial no Rio Grande do Sul. Bol. Saúde SSMA-RS, 12:2-72, 1985.

2. ALVAREZ, F. J. \& Del RIO, M. C. Decreasing prevalence of smoking in Spain [letter]. Lancet, 341:119, 1993.

3. BORHANI, N, O. Primary prevention of coronary heart disease: a critique. Am. J. Cardiol., 40:251-9, 1977.

4. CENTERS FOR DISEASE CONTROL.Cigarette smoking among adults - United States, 1990. JAMA, 267:3133. 1992.

5. CENTERS FOR DISEASECONTROL, Cigarette smoking among adults - United States, 1991. JAMA, 269:1931, 1993.

6. CONSELHO NACIONAL DE SAÚDE. Resoluça 01/88; Normas de pesquisa em saúde. Diário Oficial da Uniäo, 14 jun. 88; p. 10713-9.

7. CosTA, E. A. A cross-sectional survey of blood pressure in Rio Grande do Sul, Brazil. London, 1981. [Thesis of Doctor in Philosophy, London School of Hygiene and Tropical Medicine].

8. COSTA e SILVA, V. L. \& ROMERO, L. C. Programa nacional de combate ao fumo: plano de trabalho para o período 1988-2000. Rev. Bras. Cancerol., 34:245$54,1988$.

9. DEPARTAMENTO DE SALUD Y SERVICIOS SOCIALES DE LOS ESTADOS UNIDOS DE AMÉRICA. Tabaquismo y salud en las Américas. Atlanta, 1992 (DHHS, publicacion $n^{\circ}$ (CDC) 92-8420).

10.DUNCAN, B. B. As desigualdades sociais na distribuiç̆̃o de fatores de tisco para doenças năo-transmissiveis. Porto Alegre, 1991. [Tese de Doutorado - Universidade Federal do Rio Grande do Sul].
Tabola 6. Razōes de produtos cruzados (RPC) para fumar resultantes do modelo de regressão logistica. Porto Alegre, 1991.

\begin{tabular}{|c|c|c|c|}
\hline Condiçāo & & $\mathrm{APC}(\mathrm{IC})$ & $\mathrm{P}$ \\
\hline Sexo & $\begin{array}{l}\text { feminino" } \\
\text { masculino }\end{array}$ & $\begin{array}{c}1 \\
1,44(1,24-1,66)\end{array}$ & $<0,0001$ \\
\hline Idade & $\begin{array}{l}18-29 \text { anos } \\
30-38 \text { anos } \\
40-49 \text { anos } \\
50-88 \text { anos }\end{array}$ & $\begin{array}{c}1 \\
1,70(1,33-2,19) \\
1,00(0,78-1,29) \\
0,96(0,84-1,29)\end{array}$ & $<0,0001$ \\
\hline Renda** & $\begin{array}{l}\text { menos de } 2 \text { SH" } \\
2-3,99 \text { SH } \\
4 \text { ou mais SM }\end{array}$ & $\begin{array}{c}1 \\
0,82(0,67-0,99) \\
0,97(0,79-1,19)\end{array}$ & 0,06 \\
\hline Escolarldade & $\begin{array}{l}\text { mais de } 11 \text { anos" } \\
6-11 \text { anos } \\
0.5 \text { anos }\end{array}$ & $\begin{array}{c}1 \\
1,27(1,06-1,53) \\
0,95(0,73-1,21)\end{array}$ & 0,0399 \\
\hline Protissāo"*" & $\begin{array}{l}\text { grupo I* } \\
\text { grupo II } \\
\text { grupo III } \\
\text { gnupo IV } \\
\text { grupo V }\end{array}$ & $\begin{array}{c}1 \\
0,91(0,56-1,47) \\
1,02(0,77-1,35) \\
1,39(1,05-1,84) \\
1,21(0,90-1,62)\end{array}$ & 0,0386 \\
\hline Uso de Álcool & $\begin{array}{l}\text { nāo* } \\
\text { sim }\end{array}$ & $\begin{array}{c}1 \\
1,70(1,44-1,99)\end{array}$ & $<0,0001$ \\
\hline
\end{tabular}

- Categoria de referência

* Renda per capita, em salários-mínimos

*** Ver mátodos

11. DUNCAN, B, B.; SCHMIDT, M. I.; POLANCZYK, C. A.; HOMRICH, C. S.; ROSA, R. S.; ACHUTTI, A. C Fatores de risco para doenças não-transmissíveis em area metropolitana na regiá Sul do Brasil. prevalência e simultaneidade. Rev. Saúde Pública. 27:143-8, 1993 .

12. ESCOBEDO, A. G.; MARCUS, S. E.; HOLTZMAN, D.; GIOVINO, G. A. Sports participation, age at smoking initiation, and the risk of smoking US school students. $J A M A, 269: 1391-5,1993$

13. FOWLER, G. Smoking epidemiology. Practitioner, 235:593, 1991

14. FUCHS, F. D. Fármacos usados em cardiopatia isquêmica. In: Fuchs, F. D. \& Wannmacher, L. Farmacologia clínica: fundamentos da terapêtica racional. Rio de Janeiro, Ed. Guanabara Koogan, 1992. p. 384-409.

15. FUNDAÇẢ̄O IBGE. Censo demográfico de 1991: resultados preliminares. Porto Alegre, 1992.

16. FUNDAÇĀO IBGE. Pesquisa nacional pot amostragem domiciliar. Rio de Janeiro, 1990.

17. GUIDI. M. L. M. \& DUARTE, S. G. Un esquema de caracterização socieconômica. Rev. Bras. Estudos Pedag., 52:65-82, 1969.

18. KIRKWOOD, B. R. Essentials of medical statistics. London, Blackwell Scientific Publ., 1988.

19. KLEINBAUM, D.G.; KUPPER, C.C.; MORGENSTERN, H. Epidemiologic research, principles and quantitative methods. New York, Van Nostrand Reinhold, 1982.

20.LAURENTI, R. O problema das doenças crônicas e degenerativas e dos acidentes na Areas urbanizadas da América Latina. Rev. Saúde Pública, 9:239-48, 1975

21.LOLIO, C. A.\& LAURENTI, R. Tendenciada mortalidade por doenças cerebrovasculares em adultos maiores de 20 anos de idade na Município de Săo Paulo (Brasil), 1950 a 1981. Rev. Saúde Pública, 20:343-6, 1986. 
22. LOLIO, C. A.; SOUZA, J. M. P. de.; SANTOS A. H.; BUCHAILA, C. M. Prevalêneia de tabagismo em localidade urbana da regiāo Sudeste do Brasil. Rev. Saúdre Pública, 27:262-5, 1993.

23. MINISTÉRIO DA SAÚDE. Normas técnicas para programa nacional de controle da hipertensão arterial (PNECHA). Brasilia, 1988. (Série A: normas e manuais técnicos, 47).

24. POHLMANN, P.R.; LOSS,J,F.;FLORES, C.;BOLZZONI A.; DUNCAN, B. B.; ZIMMER, P. M. Tabagismo em Porto Alegre: Prevalência e o papel dos profíssionais da saúde na prevençāo. Rev. Ass. Med. Bras., 37:8-14, 1991.

25. POLETTO, L.; PEZzOTTO, S. M.; MORINI, J.; ANDRADE, J. Prevalência del habito de fumar en jovenes y sus padres: asociaciones relevantes con educación. Rev. Saúde Pública, 25:388-93, 1991 .
26. REGO, R. A.; BERARDO, F. A. N.; RODRIGUES, S. S. R.; OLIVEIRA, Z. M. A.; VASCONCELLOS, C.; AVENTURATO, L. V. O.: MONCAU, J. E. C.; RAMOS, L. R. Fatores de risco para doenças crônicas nāo-transmissíveis: inquérito domiciliar no Município de São Paulo, SP (Brasil): Metodologia e resultados preliminares Rev. Saúde Pública, 24;177-85, 1990.

27. RUFFINO-NETTO, A.; RUFINO, M. C.; PASSOS. A. D. C.; SILVA, C. A.; MADEIRA, A. N. Tendéncias do tabagismo entre acadêmicos de Ribeirão Preto (SP) estudo prospectivo. J. Pneumol., 14:163-9, 1988.

28. RUMEL, D. Indicadores de mortalidade por categoria ocupacional e nível socjal, Estado de São Pauło, 19801982. Såo Paulo, 1985. [Dissertaçăo de Mestrado Faculdade de Saúde Póblica da USP].

29. SIMOES, M. J. S. Tabagismo entre estudantes do primeiro e segundo graus em Araraquara, SP, Brasil, 1988. Medicina, Ribeirão Preto, 23:223-31, 1990.

\section{Abstract}

A cross-sectional study was carried out for the purpose of evaluating, the prevalence of smoking and the factors associated with it in Porto Alegre, a city in southem Brazilian. Through proportional, multiple stage, random sampling, 1.091 individuals (92\% of those eligible) of 18 or more years of age, were interviewed at home. Exposure to smoking was measured by a questionnaire that inquired about the type, quantity and frequency of tobacco use. The prevalence of smoking was $34.9 \%$ (Cl $31.9-37.8)$. It was higher -among men - 41.5\% (Cl 38.5 - 44.4) then women - $29.5 \%$ (C) 26.8 - 32.2). The former started smoking at mean age of $16( \pm 5.6)$, with mode of 15 and smoked an average of 19.0 ( \pm 14.0 ) cigarettes per day. Females started at a mean age of 17.8 ( \pm 6.7 ), with mode of 14 years old and smoked 14.5 ( \pm 10.3). The assoclation of the drinking habit and demographic and socloeconomic variables with smoking was evaluated through logistic regression. The variables included in the model were sex, age, education, income, professional qualification and alcohol consumption. The prevalence of smoking was greater for men, individuals of lower sociecinomic level, between 30 and 39 years of age, and among those accustomed to consuming alcoholic beverages. In conciusion, this study demonstrated that smoking is a public health problem in Brazil as in another countries. It is associated with $s \theta x$, age, education and professional qualification, as has been observed elsewhere. The association of alcohol consumption with smoking may be understood as risk behavior, both having similar determinants.

Smoking, epidemiology. Risk factors. 